\title{
Boundary Conditions for Three-Body Scattering in Configuration Space
}

\author{
G. L. Payne \\ Department of Physics and Astronomy, The University of Iowa, Iowa City, Iowa 52242
}

W. Glöckle

Institut für Theoretische Physik II, Ruhr-Universität Bochum, Germany

J. L. Friar

Theoretical Division, Los Alamos National Laboratory, Los Alamos, New Mexico 87545

(August 1999)

\begin{abstract}
The asymptotic behavior of three-body scattering wave functions in configuration space is studied by considering a model equation that has the same asymptotic form as the Faddeev equations. Boundary conditions for the wave function are derived, and their validity is verified by numerical calculations. It is shown that these boundary conditions for the partial differential equation can be used to obtain accurate numerical solutions for the wave function.
\end{abstract}

\section{INTRODUCTION}

In a previous paper [1] (hereafter referred to as I), we studied the asymptotic form of a three-body wave function, which results in the propagation of three free particles from various types of sources. The goal was to determine the boundary conditions appropriate for the three-body scattering equations in configuration space. In particular, we want to establish the values of 


$$
\rho=\frac{1}{2} \sqrt{\sum_{i=1}^{3}\left(\mathbf{x}_{i}-\mathbf{x}_{\mathrm{cm}}\right)^{2}}
$$

for which the leading asymptotic form of an outgoing wave would be valid and to investigate the correction terms to that form. For a complete discussion of three-body scattering in configuration space see Ref. [2], which also contains references to earlier work on this problem.

In I two sources were studied. The first was a localized source corresponding to the elastic-scattering driving term in the three-body Faddeev equation and is determined by the overlap of a two-body force in one pair and a two-body bound-state wave function in another pair. The other was an extended model source that mimics the real source term in the Faddeev equation including the breakup process in the Faddeev amplitude. The latter source reaches far out in the distance $y$ between one particle and the center of mass of the other two particles; it decreases only as $O\left(y^{-3 / 2}\right)$. However, the presence of the pair interaction limits the extent in the distance $x$ between the other two particles. As expected in the case of the extended source, the leading form is reached only at a much larger radius than the localized source, specifically when $x$ is small and $y$ is large.

By inverting the free propagator, one can determine the propagating wave function using a partial differential equation with the given source terms. We established suitable boundary conditions that could be used to solve this problem efficiently. A matching radius of about $100 \mathrm{fm}$ was found to be sufficient.

In this article we extend our previous study of the extended source to allow one pair interaction to be present while the three particles propagate from the given sources, which is exactly what happens in the Faddeev formulation. We use the same notation as in I. In Sec. II we evaluate the three-body Green's function including one pair interaction, apply it to the extended source, and study the asymptotic behavior in the two- and three-body fragmentation channels. In Sec. III we solve the related partial differential equation as an exercise for applying that technique (in a forthcoming article) to the Faddeev equation itself. Finally, we conclude in Sec. IV. 


\section{THREE-BODY PROPAGATION FROM GIVEN SOURCES WITH A PAIR INTERACTION}

In order to avoid unnecessary complications, we restricted our considerations in I to three identical bosons interacting by spin-independent s-wave pairwise interactions in a state with total angular momentum of zero. For this case the Faddeev equation for the channel with incident wave $\Phi$,

$$
\Psi=\Phi+G(E) V P \Psi
$$

where $P=P^{+}+P^{-}$is the permutation operator, reads in explicit notation as

$$
\psi(x, y)=\phi(x, y)+\int_{0}^{\infty} d x^{\prime} \int_{0}^{\infty} d y^{\prime} g\left(x, y ; x^{\prime}, y^{\prime}\right) Q\left(x^{\prime}, y^{\prime}\right)
$$

We have introduced in Eq. (2) the reduced Faddeev amplitude $\psi(x, y)$ and the corresponding reduced Green's function $g\left(x, y ; x^{\prime}, y^{\prime}\right)$. The coordinates $x$ and $y$ are the standard Jacobi variables

$$
\begin{aligned}
& \mathrm{x} \equiv \mathrm{x}_{1}=\mathrm{r}_{2}-\mathrm{r}_{3} \\
& \mathbf{y} \equiv \mathbf{y}_{1}=\mathbf{r}_{1}-\frac{1}{2}\left(\mathbf{r}_{2}+\mathbf{r}_{3}\right)
\end{aligned}
$$

expressed in terms of the individual position vectors.

The source term in Eq. (2) is given by

$$
Q(x, y)=V(x) \int_{-1}^{1} d \mu \frac{x y}{x_{2} y_{2}} \psi\left(x_{2}, y_{2}\right)
$$

where $x_{2}, y_{2}$, and $\mu$ in Eq. (5) result from (3) and (4) by the cyclical permutations, and are explicitly given by

$$
\begin{aligned}
& x_{2}=\sqrt{\frac{1}{4} x^{2}+y^{2}+x y \mu} \\
& y_{2}=\sqrt{\frac{9}{16} x^{2}+\frac{1}{4} y^{2}-\frac{3}{4} x y \mu},
\end{aligned}
$$

where $\mu$ is the cosine of the angle between $\mathbf{x}$ and $\mathbf{y}$. The total wave function is given by the sum of the three Faddeev amplitudes. In Eq. (1) $\Psi$ is one of the Faddeev amplitudes, 
the other two are generated by cyclical permutations of the particles, $P^{+} \Psi$ and $P^{-} \Psi$, and they appear in the source term as $P \Psi$. The pair interaction is $V(x)$ and this interaction also occurs in the Green's function

$$
G(E)=\frac{1}{E-\left(H_{0}+V\right)+i \varepsilon}
$$

We refer to Ref. [3] for the general background and details on the notation.

The source term has a short-range component arising from the elastic-scattering piece of the Faddeev amplitude, and a long-range component from the breakup piece of the Faddeev amplitude. In I we studied the effects of both components; however, for the long-range component we used a model source term. Using the asymptotic form of the Faddeev amplitude derived by the stationary phase approximation, one finds [2] that the asymptotic form of the source term for three equal mass particles with total energy $E$ is

$$
Q(x, y) \rightarrow C V(x) \frac{x e^{i \sqrt{4 / 3} k_{0} y}}{y^{3 / 2}},
$$

where $k_{0}^{2}=m E / \hbar^{2}$ and the constant $C$ is given by the magnitude of the wave function in the asymptotic region. Therefore, to study the effects of this long-range behavior, we used the model source term

$$
Q_{\text {Model }}(x, y)=V(x) \frac{x y e^{i \sqrt{4 / 3} k_{0} y}}{\left(y+y_{0}\right)^{5 / 2}}
$$

with $y_{0}=2 \mathrm{fm}$. This source term has the same asymptotic form as (8), and we have set $C$ to be unity for convenience.

In I we neglected the final-state interaction between one pair in the propagator. As required by the Faddeev scheme, this will now be included. We will study the second term in Eq. (2) with the source term replaced by the model source. Thus, we write

$$
F(x, y)=\int_{0}^{\infty} d x^{\prime} \int_{0}^{\infty} d y^{\prime} g\left(x, y ; x^{\prime}, y^{\prime}\right) Q_{\text {Model }}\left(x^{\prime}, y^{\prime}\right)
$$

To simplify the numerical calculations we follow the procedure used in I, and use the Bargmann two-body potential 


$$
V(x)=-\frac{V_{0} e^{-\lambda x}}{\left(1+\beta e^{-\lambda x}\right)^{2}},
$$

where

$$
V_{0}=2 \beta\left(\frac{\hbar^{2} \lambda^{2}}{M}\right)
$$

The bound-state wave function for this potential is given by

$$
u_{d}(x)=\sqrt{2 \kappa \beta} \frac{\left(1-e^{-\lambda x}\right)}{\left(1+\beta e^{-\lambda x}\right)} e^{-\kappa x},
$$

where $\kappa$ is the bound-state wave number for a two-body state with the energy $\epsilon=-\hbar^{2} \kappa^{2} / m$ and

$$
\beta=\frac{\lambda+2 \kappa}{\lambda-2 \kappa}
$$

For our model calculations we use the values $\kappa=0.2316 \mathrm{fm}^{-1}$ and $\lambda=0.7 \mathrm{fm}^{-1}$. We also need the two-body scattering states $u_{k}(x)$ for this potential. They are given by

$$
u_{k}(x)=\frac{1}{2 i}\left\{e^{i \delta(k)} e^{i k x} h(k, x)-e^{-i \delta(k)} e^{-i k x} h^{*}(k, x)\right\}
$$

with

$$
h(k, x)=\frac{1+\left(\frac{2 k-i \lambda}{2 k+i \lambda}\right) \beta e^{-\lambda x}}{1+\beta e^{-\lambda x}},
$$

and

$$
e^{i \delta(k)}=\sqrt{\frac{2 k+i \lambda}{2 k-i \lambda}} \sqrt{\frac{k+i \kappa}{k-i \kappa}} .
$$

In addition, we use the arbitrary but fixed laboratory energy of the incident particle of $E_{\text {lab }}=14 \mathrm{MeV}$ and $\hbar^{2} / m=41.47 \mathrm{MeV} \cdot \mathrm{fm}$ which corresponds to the case for three nucleons. For this case $k_{0}=0.41403 \mathrm{fm}^{-1}$. Henceforth, we set $\hbar=1$.

For three equal-mass particles with mass $m$ and total energy $E$, the three-body Green's function $g\left(x, y ; x^{\prime}, y^{\prime}\right)$ for the case with one pair interaction has the well-known form 


$$
\begin{aligned}
g\left(x, y ; x^{\prime}, y^{\prime}\right)= & u_{d}(x)\left(-\frac{4 m}{3} e^{i q_{0} y>} \frac{\sin q_{0} y_{<}}{q_{0}}\right) u_{d}\left(x^{\prime}\right) \\
& +\frac{2}{\pi} \int_{0}^{\infty} d k u_{k}(x)\left(-\frac{4 m}{3} \frac{e^{i q_{k} y_{>}} \sin q_{k} y_{<}}{q_{k}}\right) u_{k}\left(x^{\prime}\right),
\end{aligned}
$$

where $q_{0}=\sqrt{4 m / 3(E-\epsilon)}, q_{k}=\sqrt{4 / 3\left(k_{0}^{2}-k^{2}\right)}$, and $k_{0}^{2}=m E$. Obviously the propagation from the source can now proceed not only into the unbound states but also into the deuteron channel. Moreover, the two-body scattering states $u_{k}(x)$ include the interaction $V(x)$. It is the purpose of this paper to study the additional effects of $V(x)$ in the propagator, in contrast to I where only the free propagator was considered. The numerical evaluation of the second part in (11) containing $u_{k}(x)$ requires some explanation. We were not able to find an analytical expression such as we found for $g_{0}$ in I, and had to perform the $k$-integral numerically. Clearly at the upper end of the integral the integrand has rapid oscillations that make the integral difficult to evaluate numerically. Since $u_{k}(x)$ approaches $\sin k x$ as $k \rightarrow \infty$, it appears natural to subtract the free propagator $g_{0}\left(x, y ; x^{\prime}, y^{\prime}\right)$ and add it back in a separate term. Then for $k \rightarrow \infty$ the integrand has a stronger fall off and, moreover, the path of integration can be rotated into the complex plane, similar to the procedure used in Ref. [1].

Let us start with the propagation in the deuteron channel:

$$
\begin{aligned}
F_{d}(x, y) \equiv & -\frac{4 m}{3} u_{d}(x) \int_{0}^{\infty} d y^{\prime} \frac{e^{i q_{0} y_{>}} \sin q_{0} y_{<}}{q_{0}} \int_{0}^{\infty} d x^{\prime} u_{d}\left(x^{\prime}\right) Q_{\text {Model }}\left(x^{\prime}, y^{\prime}\right) \\
= & -\frac{4 m}{3} u_{d}(x) e^{i q_{0} y} \int_{0}^{\infty} d y^{\prime} \frac{\sin q_{0} y^{\prime}}{q_{0}} \int_{0}^{\infty} d x^{\prime} u_{d}\left(x^{\prime}\right) Q_{\text {Model }}\left(x^{\prime}, y^{\prime}\right) \\
& -\frac{4 m}{3} u_{d}(x) \int_{y}^{\infty} d y^{\prime} \frac{\sin q_{0}\left(y-y^{\prime}\right)}{q_{0}} \int_{0}^{\infty} d x^{\prime} u_{d}\left(x^{\prime}\right) Q_{\text {Model }}\left(x^{\prime}, y^{\prime}\right) .
\end{aligned}
$$

We see that this consists of a flux-conserving term

$$
F_{d}^{a s y}(x, y)=u_{d}(x) e^{i q_{0} y} f_{d}
$$

with

$$
f_{d}=-\frac{4 m}{3} \int_{0}^{\infty} d y^{\prime} \frac{\sin q_{0} y^{\prime}}{q_{0}} \int_{0}^{\infty} d x^{\prime} u_{d}\left(x^{\prime}\right) Q_{\text {Model }}\left(x^{\prime}, y^{\prime}\right)
$$


and a correction term

$$
F_{d}^{\text {corr }}(x, y) \equiv-\frac{4 m}{3} u_{d}(x) \int_{y}^{\infty} d y^{\prime} \frac{\sin q_{0}\left(y-y^{\prime}\right)}{q_{0}} \int_{0}^{\infty} d x^{\prime} u_{d}\left(x^{\prime}\right) Q_{\text {Model }}\left(x^{\prime}, y^{\prime}\right) .
$$

Inserting Eq. (9) into Eq. (14) and performing one partial integration in the $y^{\prime}$ variable, one arrives easily at the following asymptotic form

$$
F_{d}^{\operatorname{corr}}(x, y) \underset{y \rightarrow \infty}{\longrightarrow}-\frac{e^{i \sqrt{4 / 3} k_{0} y}}{y^{3 / 2}} u_{d}(x) \frac{1}{\epsilon} \int_{0}^{\infty} d x^{\prime} u_{d}\left(x^{\prime}\right) x^{\prime} V\left(x^{\prime}\right) .
$$

Clearly the long-range source behavior carries over into a corresponding long-range correction term in the deuteron channel. We rewrite Eq. (14) in the form

$$
F_{d}^{\text {corr }}(x, y)=\frac{e^{i \sqrt{4 / 3} k_{0} y}}{y^{3 / 2}} u_{d}(x) f_{d}^{\text {corr }}(y)
$$

and show in Fig. 1 the behavior of $f_{d}^{\text {corr }}(y)$ as it approaches its asymptotic value given by

$$
f_{d}^{\text {corr }}(y) \underset{y \rightarrow \infty}{\longrightarrow}-\frac{1}{\epsilon} \int_{0}^{\infty} d x^{\prime} u_{d}\left(x^{\prime}\right) x^{\prime} V\left(x^{\prime}\right) .
$$

To illustrate the convergence, we have normalized the plot to the asymptotic value $f_{d}^{\text {corr }}(\infty)=-23.325 \mathrm{fm}^{3 / 2}$.

To illustrate the error in the elastic term that results from matching to the asymptotic boundary conditions at a finite distance, we plot in Fig. 2 the absolute value of $f_{d}(y) \equiv$ $F_{d}(x, y) / u_{d}(x)$ and its asymptotic form given by Eq. (13) and Eq. (15). The difference is less than $2 \%$ for $y$ greater than $50 \mathrm{fm}$ and less than $1 \%$ for $y$ greater than $75 \mathrm{fm}$.

The propagation into the unbound states $u_{k}(x)$ is more complicated, since

$$
F_{\text {scat }}(x, y) \equiv-\frac{4 m}{3} \frac{2}{\pi} \int_{0}^{\infty} d k u_{k}(x) \int_{0}^{\infty} d y^{\prime} \frac{e^{i q_{k} y_{>}} \sin q_{k} y_{<}}{q_{k}} \int_{0}^{\infty} d x^{\prime} u_{k}\left(x^{\prime}\right) Q_{\text {Model }}\left(x^{\prime}, y^{\prime}\right)
$$

can be written in the form

$$
\begin{aligned}
F_{\text {scat }}(x, y)= & -\frac{4 m}{3} \frac{2}{\pi} \int_{0}^{k_{0}} d k u_{k}(x) e^{i q y} \int_{0}^{\infty} d y^{\prime} \frac{\sin q_{k} y^{\prime}}{q_{k}} \int_{0}^{\infty} d x^{\prime} u_{k}\left(x^{\prime}\right) Q_{\text {Model }}\left(x^{\prime}, y^{\prime}\right) \\
& -\frac{4 m}{3} \frac{2}{\pi} \int_{0}^{k_{0}} d k u_{k}(x) \int_{y}^{\infty} d y^{\prime} \frac{\sin q_{k}\left(y-y^{\prime}\right)}{q_{k}} \int_{0}^{\infty} d x^{\prime} u_{k}\left(x^{\prime}\right) Q_{\text {Model }}\left(x^{\prime}, y^{\prime}\right) \\
& -\frac{4 m}{3} \frac{2}{\pi} \int_{k_{0}}^{\infty} d k u_{k}(x) e^{-K y} \int_{0}^{y} d y^{\prime} \frac{\sinh K y^{\prime}}{K} \int_{0}^{\infty} d x^{\prime} u_{k}\left(x^{\prime}\right) Q_{\text {Model }}\left(x^{\prime}, y^{\prime}\right) \\
& -\frac{4 m}{3} \frac{2}{\pi} \int_{k_{0}}^{\infty} d k u_{k}(x) \frac{\sinh K y}{K} \int_{y}^{\infty} d y^{\prime} e^{-K y^{\prime}} \int_{0}^{\infty} d x^{\prime} u_{k}\left(x^{\prime}\right) Q_{\text {Model }}\left(x^{\prime}, y^{\prime}\right) \\
\equiv & F_{\text {scat }}^{(1)}(x, y)+F_{\text {scat }}^{(2)}(x, y)+F_{\text {scat }}^{(3)}(x, y)+F_{\text {scat }}^{(4)}(x, y) .
\end{aligned}
$$


In the third and fourth terms $K \equiv \sqrt{4 / 3} \sqrt{k^{2}-k_{0}^{2}}$.

Let us first examine the asymptotic behavior for fixed $x$ and $y$ approaching infinity. One has

$$
F_{\text {scat }}^{(1)}(x, y)=\int_{0}^{k_{0}} d k u_{k}(x) e^{i q_{k} y} T(k)
$$

with

$$
T(k)=-\frac{4 m}{3} \frac{2}{\pi} \int_{0}^{\infty} d y^{\prime} \frac{\sin q_{k} y^{\prime}}{q_{k}} \int_{0}^{\infty} d x^{\prime} u_{k}\left(x^{\prime}\right) Q_{\text {Model }}\left(x^{\prime}, y^{\prime}\right) .
$$

There is no saddle-point for this case; thus, the asymptotic form arises from the leading end-point contribution at $k=0$, which is easily evaluated to be

$$
\left.F_{\text {scat }}^{(1)}(x, y)\right|_{k \approx 0} \longrightarrow-\frac{\sqrt{\pi}}{4} 3^{3 / 4} k_{0}^{3 / 2} e^{i \pi / 4} \frac{e^{i \sqrt{4 / 3} k_{0} y}}{y^{3 / 2}} \tilde{u}_{0}(x) \tilde{T}_{0},
$$

where $\tilde{u}_{0}(x) \equiv u_{k}(x) /\left.k\right|_{k=0}$ and $\tilde{T}_{0} \equiv T(k) /\left.k\right|_{k=0}$. We find that

$$
\tilde{T}_{0}=-\frac{4 m}{3} \frac{2}{\pi} \int_{0}^{\infty} d y^{\prime} \frac{\sin \sqrt{\frac{4}{3}} k_{0} y^{\prime}}{\sqrt{\frac{4}{3}} k_{0}} \int_{0}^{\infty} d x^{\prime} \tilde{u}_{0}\left(x^{\prime}\right) Q_{\text {Model }}\left(x^{\prime}, y^{\prime}\right) .
$$

This term has the same dependence on $y$ as the correction term Eq. (15) in the deuteron channel. Note that $\tilde{T}_{0}$ is given by the analytical expression Eq. (22) differentiated with respect to $k$ under the integral. This is obviously true for the localized source; however, for the extended source, one must rewrite the integral using a contour deformation before performing the differentiation.

Let us now consider $F_{\text {scat }}^{(2)}(x, y)$ in Eq. (19) for the model source term given by Eq. (9)

$$
\begin{aligned}
F_{\text {scat }}^{(2)}(x, y) \longrightarrow-\frac{4 m}{3} \frac{2}{\pi} \int_{0}^{k_{0}} d k & u_{k}(x) \int_{0}^{\infty} d x^{\prime} u_{k}\left(x^{\prime}\right) x^{\prime} V\left(x^{\prime}\right) \\
& \times \int_{y}^{\infty} d y^{\prime} \frac{\sin q_{k}\left(y-y^{\prime}\right)}{q_{k}} \frac{e^{i \sqrt{4 / 3} k_{0} y^{\prime}}}{y^{\prime 3 / 2}} .
\end{aligned}
$$

After one partial integration one finds

$$
F_{\text {scat }}^{(2)}(x, y) \underset{\substack{y \rightarrow \infty \\ x \text { fixed }}}{\longrightarrow}-\frac{2}{\pi} \int_{0}^{k_{0}} d k u_{k}(x) \frac{m}{k^{2}} \int_{0}^{\infty} d x^{\prime} u_{k}\left(x^{\prime}\right) x^{\prime} V\left(x^{\prime}\right) \frac{e^{i \sqrt{4 / 3} k_{0} y}}{y^{3 / 2}}
$$


The term $F_{\text {scat }}^{(3)}(x, y)$, as it stands, is less obvious in its asymptotic behavior. The contributions to the $y^{\prime}$-integral keep growing towards the upper limit $y$. Because of the factor $\exp (-K y)$, only contributions from the upper end of the $y^{\prime}$ integral have to be considered. Again by partial integration, one easily finds

$$
\begin{aligned}
F_{\text {scat }}^{(3)}(x, y) \underset{\substack{y \rightarrow \infty \\
x \text { fixed }}}{\longrightarrow}-\frac{4 m}{3} \frac{2}{\pi} \frac{e^{i \sqrt{4 / 3} k_{0} y}}{y^{3 / 2}} \int_{k_{0}}^{\infty} d k & u_{k}(x) \frac{1}{2 K} \frac{1}{K+i \sqrt{4 / 3} k_{0}} \\
& \times \int_{0}^{\infty} d x^{\prime} u_{k}\left(x^{\prime}\right) x^{\prime} V\left(x^{\prime}\right) .
\end{aligned}
$$

Finally, the last piece, $F_{\text {scat }}^{(4)}(x, y)$, can again be handled in a straightforward manner with the result

$$
\begin{aligned}
F_{\text {scat }}^{(4)}(x, y) \underset{\substack{y \rightarrow \infty \\
x \text { fixed }}}{\longrightarrow}-\frac{4 m}{3} \frac{2}{\pi} \frac{e^{i \sqrt{4 / 3} k_{0} y}}{y^{3 / 2}} \int_{k_{0}}^{\infty} d k & u_{k}(x) \frac{1}{2 K} \frac{1}{K-i \sqrt{4 / 3} k_{0}} \\
& \times \int_{0}^{\infty} d x^{\prime} u_{k}\left(x^{\prime}\right) x^{\prime} V\left(x^{\prime}\right) .
\end{aligned}
$$

Adding equations (26), (27), and (28) we obtain the concise result given in Ref. [5],

$$
F_{\text {scat }}^{(2)}+F_{\text {scat }}^{(3)}+F_{\text {scat }}^{(4)} \longrightarrow \frac{2}{\pi} \frac{e^{i \sqrt{4 / 3} k_{0} y}}{y^{3 / 2}} \int_{0}^{\infty} d k u_{k}(x) \frac{m}{k^{2}} \int_{0}^{\infty} d x^{\prime} u_{k}\left(x^{\prime}\right) x^{\prime} V\left(x^{\prime}\right)
$$

This can be simplified by using a technique suggested by C. Gignoux [6]. Writing the two-body Green's function in the form

$$
g_{2}\left(x, x^{\prime} ; z\right) \equiv u_{d}(x) \frac{1}{z-\epsilon} u_{d}\left(x^{\prime}\right)+\frac{2}{\pi} \int_{0}^{\infty} d k u_{k}(x) \frac{1}{z-k^{2} / m} u_{k}\left(x^{\prime}\right)
$$

the integral over $k$ occurring in Eq. (29) can be rewritten as

$$
-\frac{2}{\pi} \int_{0}^{\infty} d k u_{k}(x) \frac{m}{k^{2}} u_{k}\left(x^{\prime}\right)=g_{2}\left(x, x^{\prime} ; 0\right)+u_{d}(x) \frac{1}{\epsilon} u_{d}\left(x^{\prime}\right) \text {. }
$$

Thus, we are led to the function

$$
\tilde{u}(x) \equiv \int_{0}^{\infty} d x^{\prime} g_{2}\left(x, x^{\prime} ; 0\right) x^{\prime} V\left(x^{\prime}\right)
$$

which obeys the inhomogeneous equation 


$$
\left[-\frac{1}{m} \frac{d^{2}}{d x^{2}}+V(x)\right] \tilde{u}(x)=-x V(x)
$$

Using the explicit form for $g_{2}\left(x, x^{\prime} ; 0\right)$, one easily derives

$$
\tilde{u}(x) \underset{x \rightarrow \infty}{\longrightarrow}-\int_{0}^{\infty} d x^{\prime} \tilde{u}_{0}\left(x^{\prime}\right) x^{\prime} V\left(x^{\prime}\right)=a
$$

where $a$ is the scattering length defined by $\delta(k)$ approaching $-k a$ as $k$ goes to zero. From Eq. (33) and Eq. (34) follows

$$
\tilde{u}(x)=\tilde{u}_{0}(x)-x .
$$

We now have the concise form

$$
F_{\text {scat }}^{(2)}+F_{\text {scat }}^{(3)}+F_{\text {scat }}^{(4)} \underset{\substack{y \rightarrow \infty \\ x \text { fixed }}}{\longrightarrow} \frac{e^{i \sqrt{4 / 3} k_{0} y}}{y^{3 / 2}}\left[\tilde{u}(x)+u_{d}(x) \frac{1}{\epsilon} \int_{0}^{\infty} d x^{\prime} u_{d}\left(x^{\prime}\right) x^{\prime} V\left(x^{\prime}\right)\right] .
$$

Altogether $F_{\text {scat }}(x, y)$ has the asymptotic form

$$
\begin{array}{r}
F_{\text {scat }}(x, y) \underset{\substack { y \rightarrow \infty \\
\begin{subarray}{c}{x \text { fixed } \\
\rightarrow{ y \rightarrow \infty \\
\begin{subarray} { c } { x \text { fixed } \\
\rightarrow } }\end{subarray}}{\longrightarrow} \frac{e^{i \sqrt{4 / 3} k_{0} y}}{y^{3 / 2}}\left[-\tilde{u}_{0}(x) \sqrt{\frac{\pi}{4}} 3^{3 / 4} k_{0}^{3 / 2} e^{i \pi / 4} \tilde{T}_{0}+\tilde{u}_{0}(x)-x\right. \\
\left.+u_{d}(x) \frac{1}{\epsilon} \int_{0}^{\infty} d x^{\prime} u_{d}\left(x^{\prime}\right) x^{\prime} V\left(x^{\prime}\right)\right] .
\end{array}
$$

The $x$-dependence is therefore built up of the zero-energy scattering state, a linear term in $x$, and the two-body bound state. The last term cancels exactly against the correction term Eq. (15) in the deuteron channel and the total amplitude $F(x, y)$ behaves as

$$
F(x, y) \underset{\substack{y \rightarrow \infty \\ x \text { fixed }}}{\longrightarrow} u_{d}(x) e^{i q_{0} y} f_{d}+\frac{e^{i \sqrt{4 / 3} k_{0} y}}{y^{3 / 2}}\left[-\tilde{u}_{0}(x) \sqrt{\frac{\pi}{4}} 3^{3 / 4} k_{0}^{3 / 2} e^{i \pi / 4} \tilde{T}_{0}+\tilde{u}_{0}(x)-x\right] .
$$

For $x$ outside the range of $V(x)$, the expression in the brackets in Eq. (38) reduces to

$$
-(x-a) \frac{\sqrt{\pi}}{4} 3^{3 / 4} k_{0}^{3 / 2} e^{i \pi / 4} \tilde{T}_{0}-a .
$$

\footnotetext{
${ }^{1}$ An alternate derivation of this result is given in Section 6.3 of Ref. [2]; however, the solution $g(x)=-1$ of Eq. (2.6.19) in Ref. [2] is not given explicitly.
} 
To verify the validity of the asymptotic term in Eq. (37), we use Eq. (10) to numerically evaluate $F(x, y)$ for several values of $y$ using only the breakup component of the Green's function given in Eq. (11). We show the convergence to the asymptotic result by rewriting $F_{\text {scat }}(x, y)$ in the form

$$
F_{\text {scat }}(x, y)=\frac{e^{i \sqrt{4 / 3} k_{0} y}}{y^{3 / 2}} a(x, y)
$$

where from Eq. (37) $a(x, y)$ has the asymptotic form

$$
-\tilde{u}_{0}(x) \sqrt{\frac{\pi}{4}} 3^{3 / 4} k_{0}^{3 / 2} e^{i \pi / 4} \tilde{T}_{0}+\tilde{u}(x)+u_{d}(x) \frac{1}{\epsilon} \int_{0}^{\infty} d x^{\prime} u_{d}\left(x^{\prime}\right) x^{\prime} V\left(x^{\prime}\right) .
$$

The results for several values of $y$ and the asymptotic form are shown in Fig. 3, where one can see that $a(x, y)$ approaches its asymptotic form for large values of $y$. To better illustrate the convergence, in Fig. 4 we plot $a(x, y)$ versus $1 / y$ for $x=3$. As $1 / y$ goes to zero the plot approaches its asymptotic value $a(3, \infty)=6.863-4.005 i$ given by Eq. (41).

Let us now regard the asymptotic form of $F_{\text {scat }}(x, y)$ for both $x$ and $y$ going to infinity at a certain fixed angle $\theta$ in the first quadrant. For this case only the breakup part contributes. Its first term $F_{\text {scat }}^{(1)}$ receives contributions from a saddle point and from the two end points. The result is

$$
F_{\text {scat }}(x, y) \longrightarrow \frac{e^{i k_{0} \rho}}{\left(k_{0} \rho\right)^{1 / 2}}\left[A(\theta)+\frac{1}{k_{0} \rho} B(\theta)+\cdots\right]
$$

where $\theta$ and $\rho$ are defined by $x=\rho \cos \theta, y=\sqrt{3 / 4} \rho \sin \theta$, and

$$
\begin{gathered}
A(\theta)=-k_{0} e^{i \pi / 4} \sqrt{\frac{\pi}{2}} \sin \theta e^{i \delta\left(k_{0} \cos \theta\right)} T\left(k_{0} \cos \theta\right), \\
B(\theta)=\frac{1}{2 i}\left[\frac{1}{4} A(\theta)+A^{\prime \prime}(\theta)\right] .
\end{gathered}
$$

In the following discussion we use $(x, y)$ and $(\rho, \theta)$ interchangeably. The end-point contributions are of $O\left(\rho^{-2}\right)$ and are beyond what is displayed in Eq. (42). The relation between $B(\theta)$ and $A(\theta)$ is the same as found for the free propagation case considered in I. A tedious analytical study reveals that for this case the only contributions up to order $\rho^{-3 / 2}$ are from 
$F_{\text {scat }}^{(1)}$. Thus the two leading terms in Eq. (42) result solely from $F_{\text {scat }}^{(1)}$ in Eq. (20). The same is of course true for the free propagator $g_{0}$ studied in I.

It is clear that the first term alone in Eq. (42), the flux-conserving breakup behavior, is not a valid representation of $F_{\text {scat }}$ at small $\rho$ values. The correction term is suppressed only by $O\left[\left(k_{0} \rho\right)^{-1}\right]$, and depending upon the size of $B(\theta)$ relative to $A(\theta)$ the value of $\rho$ may have to be very large before one can neglect the second- and higher-order terms in Eq. (42). To illustrate this property we numerically evaluate Eq. (21) at $\rho_{\mathrm{m}}$ and $\rho_{\mathrm{m}} \pm 10 \mathrm{fm}$ for a fixed value of $\theta$, which is then fit to the function

$$
\left[a_{\mathrm{m}}+\frac{b_{\mathrm{m}}}{k_{0} \rho}+\frac{c_{\mathrm{m}}}{\left(k_{0} \rho\right)^{2}}\right] \frac{e^{i k_{0} \rho}}{\left(k_{0} \rho\right)^{1 / 2}} .
$$

The $a_{\mathrm{m}}$ for three values of $\theta$ along with the asymptotic value $A(\theta)$ are given in Table I.

From Table I one can see that Eq. (45) provides an accurate approximation to $F_{\text {scatt }}(x, y)$ at reasonable values of $\rho$. In addition, we note that the value of $\rho_{\mathrm{m}}$ required for convergence increases as $\theta$ increases. This feature is due to the property that large values of $\theta$ correspond to small values of $x$, and for $x$ small one must use Eq. (37). From the Taylor-series expansion in $x$ of Eq. (37) one finds that for $x$ greater than the range of the bound state and $(x / \rho)^{2}$ small

$$
F_{\text {scat }}(x, y) \longrightarrow A(\theta) \frac{e^{i k_{0} \rho}}{\left(k_{0} \rho\right)^{1 / 2}} .
$$

For $\theta=80^{\circ}$ the value of $\rho$ must be larger than $200 \mathrm{fm}$ for this approximation to be valid.

\section{THE PARTIAL-DIFFERENTIAL EQUATION APPROACH}

To test the accuracy of solving the differential form of the Faddeev equations in configuration space we solve the partial differential equation

$$
g^{-1} F=Q
$$

which has the explicit form 


$$
\left[-\frac{1}{m}\left(\frac{\partial^{2}}{\partial \rho^{2}}+\frac{1}{\rho} \frac{\partial}{\partial \rho}+\frac{1}{\rho^{2}} \frac{\partial^{2}}{\partial \theta^{2}}\right)+V(\rho \cos \theta)-E\right] F(\rho, \theta)=-Q(\rho, \theta),
$$

and impose boundary conditions along a quarter circle in the first $x-y$ quadrant at $\rho=\rho_{\max }$. To solve the partial differential equation, we write

$$
F(\rho, \theta) \equiv \frac{e^{i k_{0} \rho}}{\left(k_{0} \rho+\beta\right)^{1 / 2}} f(\rho, \theta)
$$

and solve the resulting partial differential equation for $f(\rho, \theta)$. The constant $\beta$ is a parameter introduced to avoid singular behavior at the origin. We solved this equation using the spline expansion methods described in [7] for $\rho<\rho_{\max }$ with the boundary condition that $F(\rho, \theta)$ have the form at $\rho_{\max }$ specified by Eq. (45).

To show that this procedure can be used to obtain accurate results for the scattering wave function, we solved the partial differential equation numerically for various values of $\rho_{\max }$ and compared the results to those obtained by numerically integrating the Green's function integral in Eq. (21). We found that values of $\rho_{\max }$ on the order of $100 \mathrm{fm}$ can be used to obtain good wave functions. Larger values of $\rho_{\max }$ yield more accurate solutions. In Figs. 5 and 6 we have plotted examples of the comparisons for fixed $\rho$ values of $25 \mathrm{fm}$ and $50 \mathrm{fm}$. One can see that in both cases the agreement is excellent.

For the three-body scattering problem one wants to obtain accurate values of the breakup amplitude $A(\theta)$. Since this corresponds to the amplitude of the scattering wave function at infinity, its value cannot be obtained by evaluating the Faddeev amplitude at large values of $\rho_{\max }$. In Fig. 7 we compare the "exact" result for $A(\theta)$ evaluated using Eq. (43) and the integral form for $T(k)$ given in Eq. (22) to the values extracted from the wave function evaluated at $\rho_{\max }=200 \mathrm{fm}$. To demonstrate again that the boundary conditions for the partial differential equation have been treated correctly, we show the $A(\theta)$ extracted from the numerical solution of the Faddeev equation and the evaluation of the Green's function integral with $\rho=\rho_{\max }$. While the wave function results are similar to the "exact" values, one can see that for large values of $\theta$ they are different for the reasons discussed in the previous section. The $A(\theta)$ obtained from the wave function at $\rho_{\max }$ still exhibits the small 
$x$ behavior of the wave function for $\theta$ near $90^{\circ}$. Thus, to obtain accurate results for $A(\theta)$ one must use the integral expression. This was the procedure followed in Ref. [8] where it was shown that configuration-space Faddeev calculations gave results in excellent agreement with the momentum-space calculations.

\section{CONCLUSIONS}

Using a model source that mimics the real source term in the configuration-space Faddeev equations, the validity of the expressions for the asymptotic behavior of the wave function has been verified by numerically integrating the integral representation of the Green's function integral. Using the asymptotic expressions as the boundary conditions for the partial differential equation in configuration space, it is possible to obtain an accurate solution of the scattering equation for reasonable values of $\rho_{\max }$. While larger values of $\rho_{\max }$ are required to obtain more accurate solutions, values on the order of $100 \mathrm{fm}$ yield good solutions. The breakup amplitude corresponding to small values of $x$ cannot be obtained from the wave

function evaluated at large values of $\rho$; the T-matrix integral must be used to determine $A(\theta)$ in this region.

\section{ACKNOWLEDGMENTS}

The work of J. L. F. was performed under the auspices of the U. S. Department of Energy. That of G. L. P. was supported in part by the U. S. Department of Energy. 


\section{REFERENCES}

[1] W. Glöckle and G. L. Payne, Phys. Rev. C 45, 974 (1992).

[2] J. L. Friar and G. L. Payne, "Proton-Deuteron Scattering and Reactions," in Coulomb Interactions in Nuclear and Atomic Few-Body Collisions, ed. by F. S. Levin and D. A. Micha, (Plenum Press, New York, 1996), p. 97.

[3] W. Glöckle, The Quantum Mechanical Few-Body Problem (Springer-Verlag, Berlin, 1983).

[4] W. Glöckle, Phys. Rev. C 37, 6 (1988).

[5] W. Glöckle, Z. f. Physik 271, 31 (1974).

[6] C. Gignoux, private communication.

[7] G. L. Payne, "Configuration-Space Faddeev Calculations: Numerical Methods," in Models and Methods in Few-Body Physics, ed. by L. S. Ferreira, A. C. Fonseca, and L. Streit, (Springer-Verlag, Berlin Heidelberg, 1987) p. 64.

[8] J. L. Friar, G. L. Payne, W. Glöckle, D. Hüber, and H. Witała, Phys. Rev. C 51, 2356 (1995). 
Table I: Values of $a_{\mathrm{m}}$ determined from Eq. (45) for $\theta=30^{\circ}, \theta=60^{\circ}$, and $\theta=80^{\circ}$. The values for $\rho_{\mathrm{m}}=\infty$ were determined from Eq. (43).

\begin{tabular}{|r|c|c|c|c|cc|}
\hline$\rho_{\mathrm{m}}$ & \multicolumn{2}{|c|}{$80^{\circ}$} & \multicolumn{2}{c|}{$60^{\circ}$} & \multicolumn{2}{c|}{$30^{\circ}$} \\
\hline \hline & Real & Imag & Real & Imag & Real & Imag \\
\cline { 2 - 7 } 50.0 & -0.2112 & 0.1240 & 0.0595 & 0.2159 & 0.0173 & 0.0657 \\
90.0 & -0.1088 & 0.1718 & 0.0137 & 0.1921 & 0.0152 & 0.0642 \\
140.0 & -0.0737 & 0.1651 & 0.0083 & 0.1969 & 0.0155 & 0.0642 \\
190.0 & -0.0678 & 0.1609 & 0.0074 & 0.1980 & 0.0156 & 0.0642 \\
240.0 & -0.0665 & 0.1586 & 0.0070 & 0.1984 & 0.0157 & 0.0642 \\
290.0 & -0.0660 & 0.1578 & 0.0069 & 0.1986 & 0.0157 & 0.0642 \\
340.0 & -0.0659 & 0.1574 & 0.0068 & 0.1988 & 0.0157 & 0.0642 \\
$\infty$ & -0.0657 & 0.1560 & 0.0066 & 0.1990 & 0.0157 & 0.0642 \\
\hline
\end{tabular}




\section{FIGURE CAPTIONS}

Figure 1. The behavior of $f_{d}^{\text {corr }}(y)$ normalized to its asymptotic value for $y \rightarrow \infty$.

Figure 2. Comparison of the absolute value of $f_{d}(y)$ to its asymptotic form.

Figure 3. The $x$ dependence of $a(x, y)$ defined in Eq. (40) for fixed values of $y$ and its asymptotic form.

Figure 4 . The real and imaginary parts of $a(x, y)$ for $x=3$ plotted versus $1 / y$. The triangles are the calculated values and the solid line is a fit to a polynomial in $1 / y$.

Figure 5. Comparison of the $f(\rho, \theta)$ evaluated using the Green's function integral and the $f(\rho, \theta)$ obtained from solving the partial differential equation for $\rho=25 \mathrm{fm}$.

Figure 6. Same as for Fig. 5 for $\rho=50 \mathrm{fm}$.

Figure 7. Comparison of the breakup amplitude, $A(\theta)$, evaluated using the integral form for $T(k)$ with the $A(\theta)$ extracted from the wave function at $\rho_{\max }=200 \mathrm{fm}$. Wave function results for both the numerical solution of the Faddeev equation and the Green's function integral are shown. 
FIGURES 
FIGURE 1

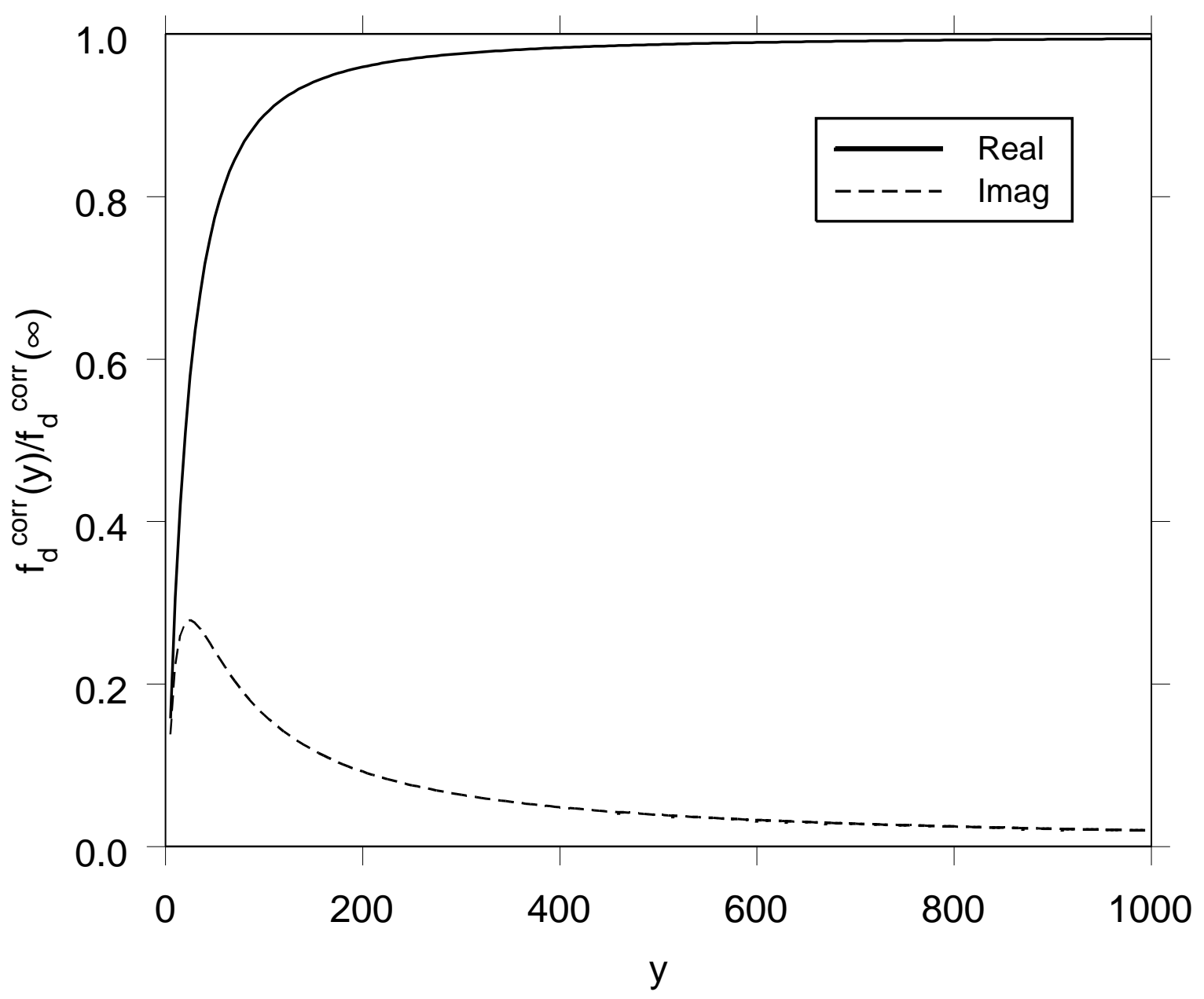


FIGURE 2

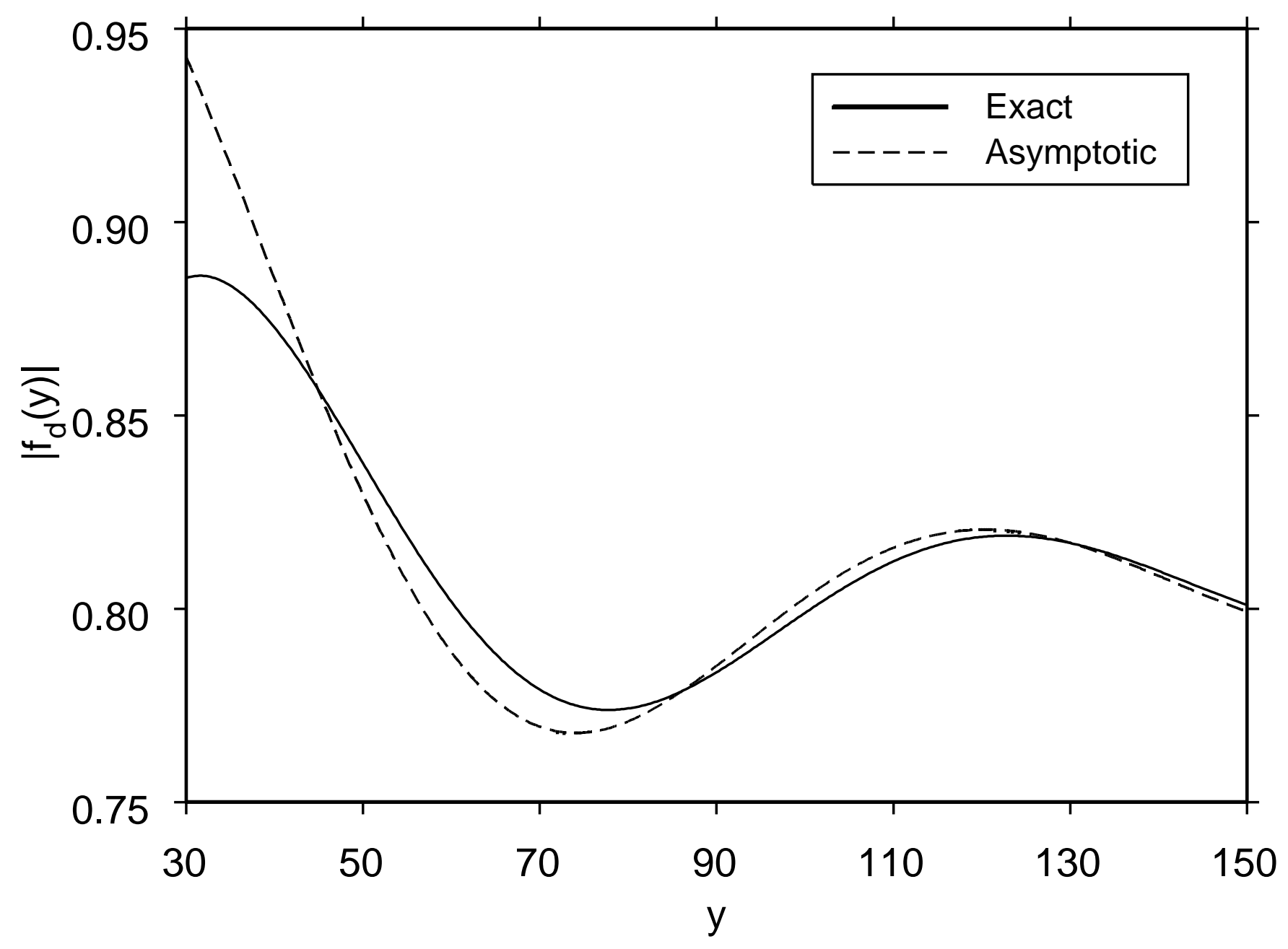


FIGURE 3
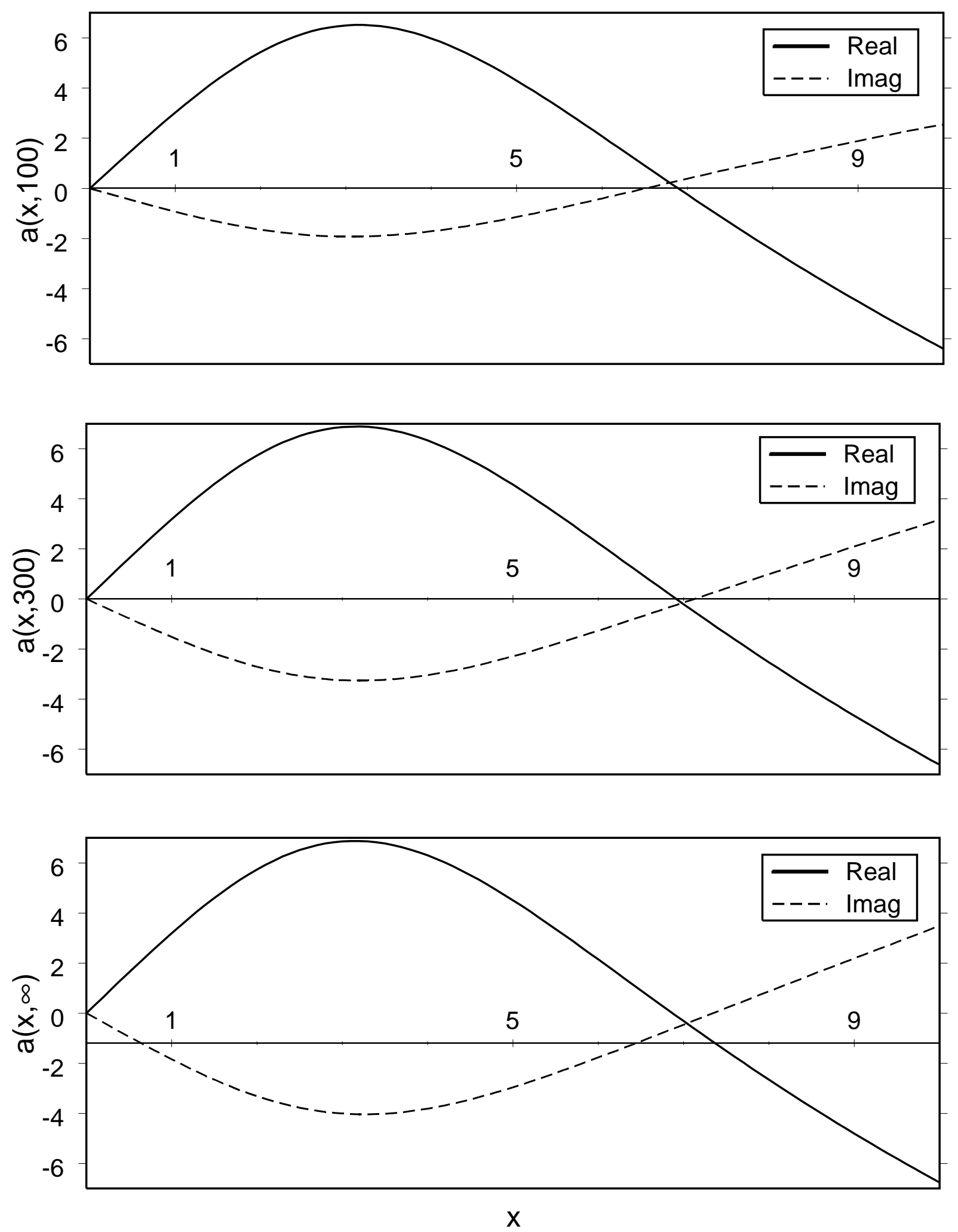
FIGURE 4
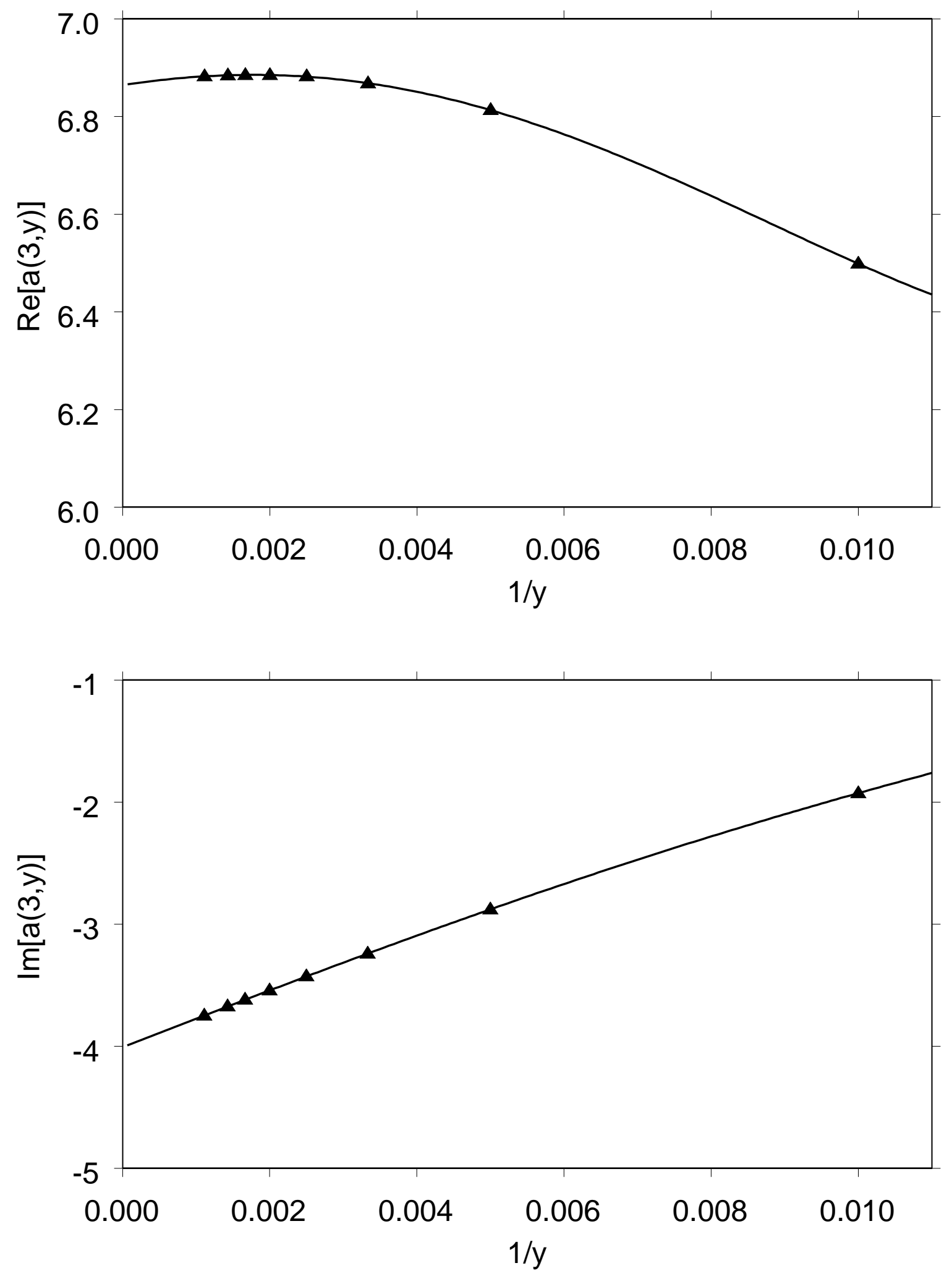
FIGURE 5
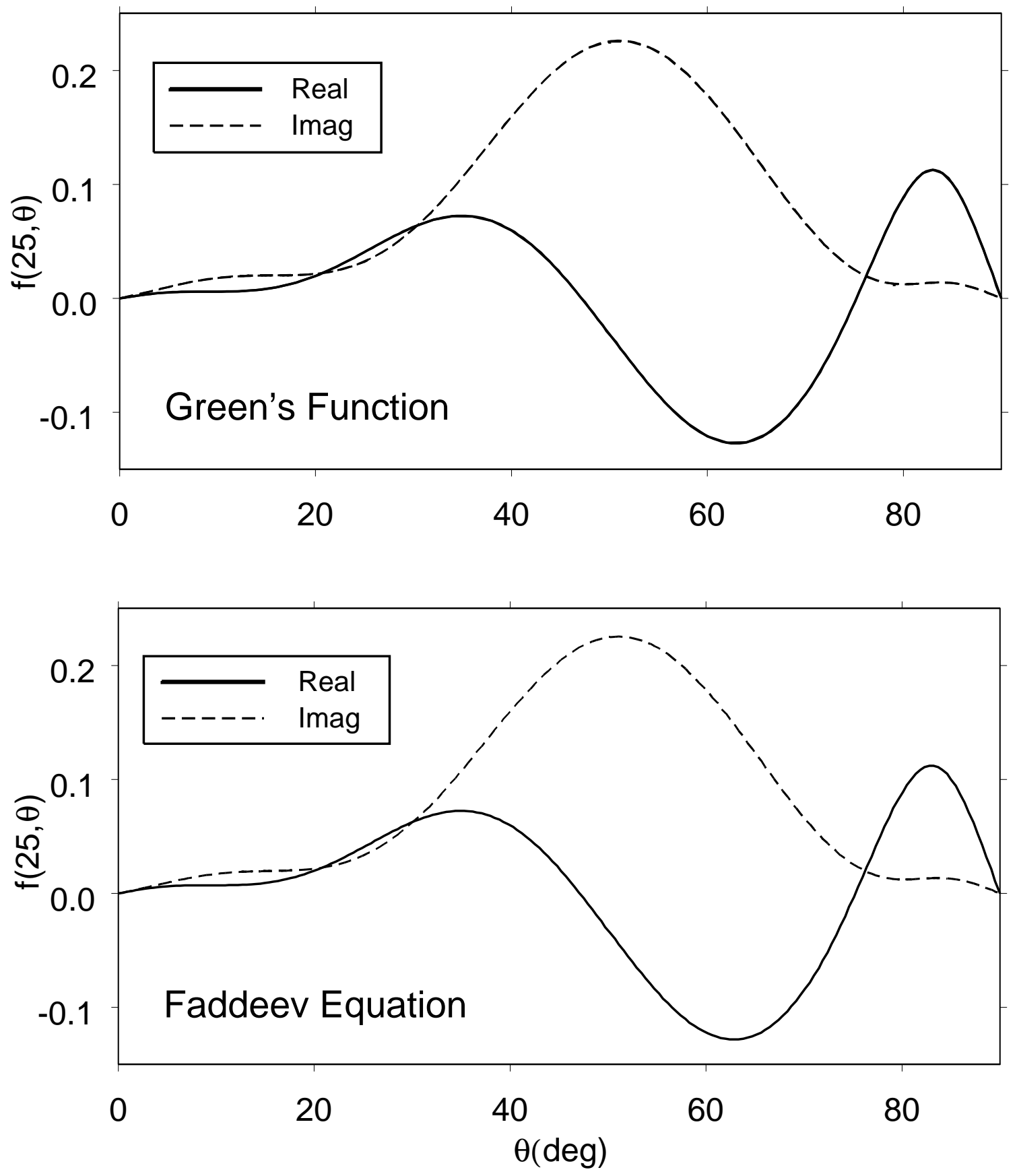
FIGURE 6
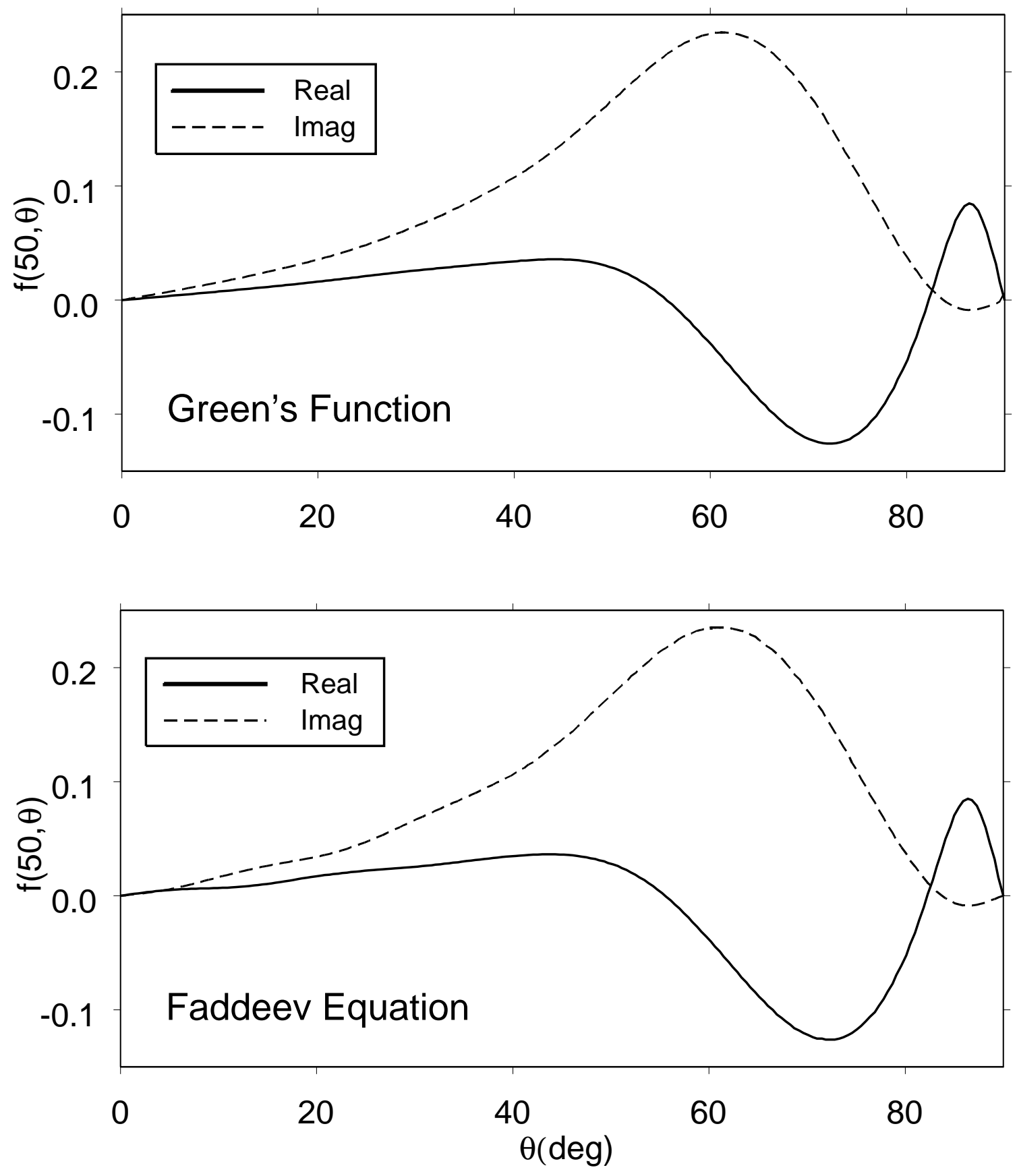
FIGURE 7
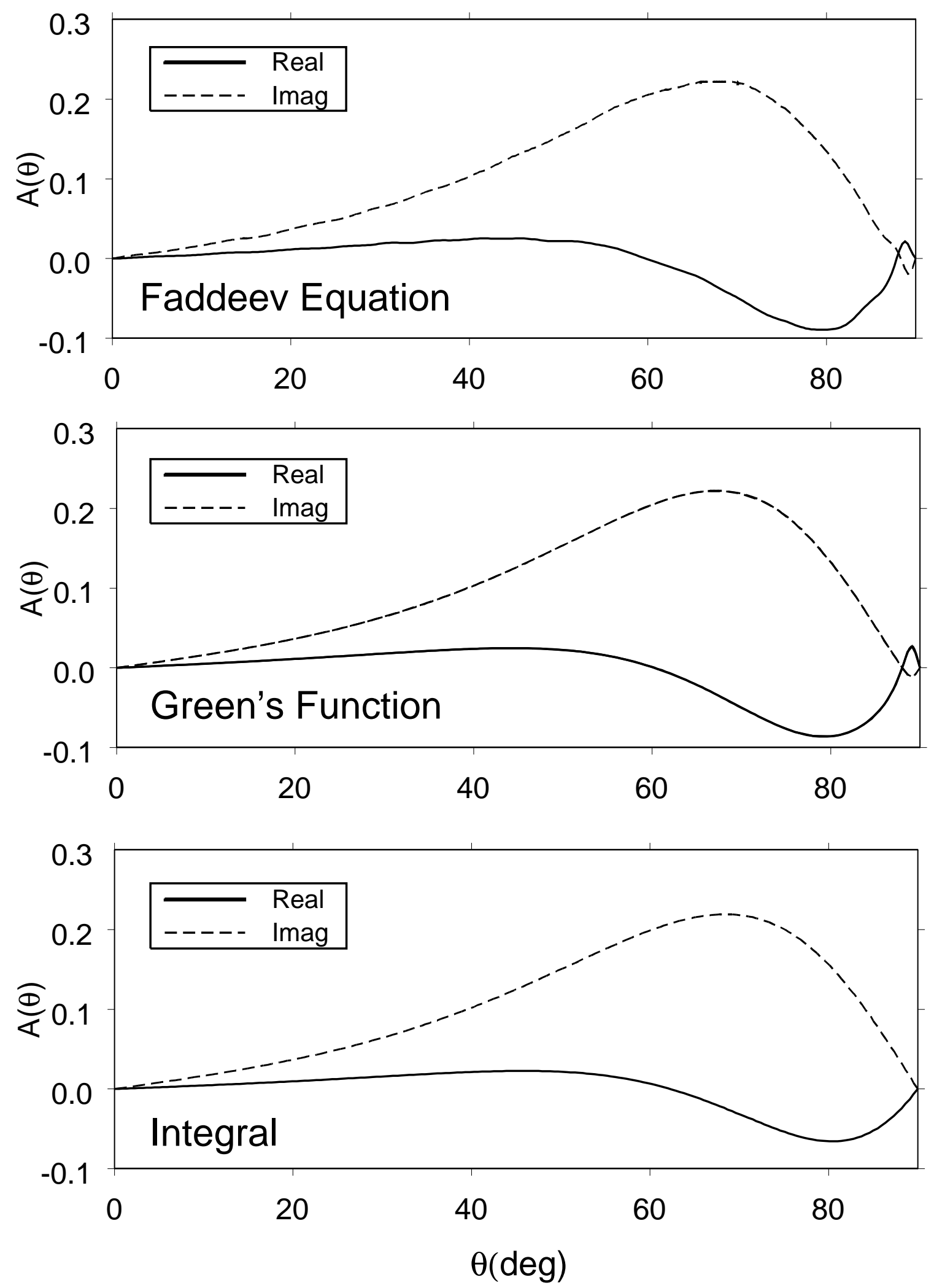